\title{
Phenomenologies of exotic particles in the lepton sector
}

\author{
Sandy S. C. Law* \\ Department of Physics, \\ Chung Yuan Christian University, \\ Chung-Li 32023, Taiwan \\ E-mail: SLawdeycu.edu.tw
}

\section{Chun-Khiang Chua}

Department of Physics,

Chung Yuan Christian University,

Chung-Li 32023, Taiwan

E-mail: ckchuadcycu.edu.tw

\begin{abstract}
By considering all new interaction terms in the lepton sector that are renormalizable and SM gauge invariant, but with only one exotic particle appearing per term, we have identified that the phenomenologies of a set of new triplet leptons (with nonzero hypercharge) coming from such minimal couplings has been rarely studied. In this work, we aim to study their contributions to the FCNC effects, and using available low-energy experimental data to place bounds on the relevant parameters associated with these new triplets.
\end{abstract}

35th International Conference of High Energy Physics

July 22-28, 2010

Paris, France

${ }^{*}$ Speaker. 


\section{Introduction}

The discovery of neutrino oscillations has long been suggestive of new physics in the lepton sector. It provides compelling evidence for nonzero neutrino masses, and hints of possible lepton flavor violation (LFV). In this work, we consider the following minimal coupling as a simple way to introduce new physics to the lepton sector:

$$
Y_{\text {exotic }}(\mathrm{SM} \text { particle }) \cdot(\mathrm{SM} \text { particle }) \cdot(\text { exotic particle }) \text {. }
$$

Imposing SM gauge and Lorentz symmetries, we note that many of the exotic particles induced this way have been studied before due to other motivations. Of the ones that have been rarely discussed, we study the exotic lepton triplets, $E_{L, R}=\left(E_{L, R}^{0}, E_{L, R}^{-}, E_{L, R}^{--}\right)^{T}$, which transform like $(1,3,-1)$ under the SM gauge groups (with $Q=I_{3}+Y$ ).

\section{The Model Setup}

To analyse the new sources of FCNC contribution from these triplets, we write them as

$$
E_{R, L}=\left(\begin{array}{cc}
E_{R, L}^{-} / \sqrt{2} & E_{R, L}^{0} \\
E_{R, L}^{--} & -E_{R, L}^{-} / \sqrt{2}
\end{array}\right),
$$

while the interaction Lagrangian of interest is given by

$$
\mathscr{L}^{E}=\operatorname{Tr}\left[\bar{E}_{R, L} i \not D E_{R, L}\right]-\operatorname{Tr}\left[\bar{E}_{R} M_{E} E_{L}+\text { h.c. }\right]-\left[\bar{L}_{L} Y_{E} E_{R} \phi+\bar{L}_{L} Y_{\ell} \phi \ell_{R}+\bar{L}_{L} Y_{v} \phi^{c} v_{R}+\text { h.c. }\right],
$$

where $L_{L}=\left(v_{L}, \ell_{L}\right)^{T}$ is the usual lepton doublet and we have included the RH neutrinos, $v_{R} \sim$ $(1,1,0)$ to give neutrino a Dirac mass. To study the mixing between SM leptons and components of the exotic triplets, it is convenient to package the mass terms as follows

$$
\mathscr{L}^{\text {mass }}=-\left(\bar{v}_{R} \overline{E_{R}^{0}}\right)\left(\begin{array}{cc}
m_{D}^{\dagger} & 0 \\
v Y_{E}^{\dagger} / \sqrt{2} & M_{E}
\end{array}\right)\left(\begin{array}{c}
v_{L} \\
E_{L}^{0}
\end{array}\right)-\left(\bar{\ell}_{R} \overline{E_{R}^{-}}\right)\left(\begin{array}{cc}
m_{\ell} & 0 \\
-v Y_{E}^{\dagger} / 2 & M_{E}
\end{array}\right)\left(\begin{array}{c}
\ell_{L} \\
E_{L}^{-}
\end{array}\right)+\text {h.c. . }
$$

where $v$ denotes the Higgs VEV. The diagonalization process that put these fields into their mass eigenbasis will give rise to new generalised coupling matrices for the charged and neutral currents which contain new sources of LFV (see [四] for details). We parameterize this by defining the dimensionless quantity:

$$
\lambda \equiv \frac{v^{2}}{8} Y_{E} \frac{1}{M_{E}^{2}} Y_{E}^{\dagger}
$$

where we observe that new contributions to tree-level flavor changing currents would be provided by the nonzero off-diagonal entries of matrix $\lambda$.

\section{Constraints from experiments}

In this section, we present the constraints on the elements of $\lambda$ from various leptonic processes. Since the off-diagonal values are the sources for LFV, studying processes such as charged lepton 
decays (e.g. $\ell \rightarrow 3 \ell^{\prime}$ and $\ell \rightarrow \ell^{\prime} \gamma$ ) or $\mu$-e conversion in atomic nuclei will place stringent bounds on $\lambda_{\alpha \beta}(\alpha \neq \beta)$, whereas the diagonal elements can be examined by looking into $Z \rightarrow \ell_{\alpha} \bar{\ell}_{\alpha}$.

It should be noted that because there is new tree-level FCNC, the Fermi constant as extracted from muon decay is now modified and is related to the $\mathrm{SM}$ version of $G_{F}$ via:

$$
G_{F}^{\prime} \simeq G_{F} \sqrt{1-2 \lambda_{e e}-2 \lambda_{\mu \mu}} \text {, with } \quad G_{F}^{2} \equiv g^{4} / 32 M_{W}^{4} .
$$

Using the experimental data [ [] from the various processes mentioned, we obtain the following summary of constraints on the elements of $\lambda$ :

\begin{tabular}{|c|c|c|}
\hline parameter(s) & process & constraint \\
\hline$\lambda_{e e}$ & $Z \rightarrow e^{-} e^{+}$ & $-2.7 \mp 0.4 \times 10^{-3}$ \\
$\lambda_{\mu \mu}$ & $Z \rightarrow \mu^{-} \mu^{+}$ & $-2.9 \mp 0.4 \times 10^{-3}$ \\
$\lambda_{\tau \tau}$ & $Z \rightarrow \tau^{-} \tau^{+}$ & $-3.1 \mp 0.4 \times 10^{-3}$ \\
\hline & $Z \rightarrow e^{ \pm} \mu^{\mp}$ & $<1.8 \times 10^{-3}$ \\
$\left|\lambda_{e \mu}\right|$ & $\mu^{-} \rightarrow e^{-} e^{-} e^{+}$ & $<1.1 \times 10^{-6}$ \\
& $\mu \rightarrow e \gamma$ & $<2.2 \times 10^{-4}$ \\
& $\mu-e$ conversion in Ti & $<5.3 \times 10^{-7}$ \\
\hline & $Z \rightarrow e^{ \pm} \tau^{\mp}$ & $<4.3 \times 10^{-3}$ \\
$\left|\lambda_{e \tau}\right|$ & $\tau^{-} \rightarrow e^{-} e^{-} e^{+}$ & $<5.0 \times 10^{-4}$ \\
& $\tau^{-} \rightarrow e^{-} \mu^{-} \mu^{+}$ & $<6.5 \times 10^{-4}$ \\
& $\tau \rightarrow e \gamma$ & $<2.7 \times 10^{-2}$ \\
\hline$\left|\lambda_{\mu \tau}\right|$ & $Z \rightarrow \mu^{ \pm} \tau^{\mp}$ & $<4.7 \times 10^{-3}$ \\
& $\tau^{-} \rightarrow \mu^{-} \mu^{-} \mu^{+}$ & $<4.8 \times 10^{-4}$ \\
& $\tau^{-} \rightarrow \mu^{-} e^{-} e^{+}$ & $<5.5 \times 10^{-4}$ \\
$\left|\lambda_{\mu e}\right|\left|\lambda_{\mu \tau}\right|$ & $\tau \rightarrow \mu \gamma$ & $<3.1 \times 10^{-2}$ \\
$\left|\lambda_{e \mu}\right|\left|\lambda_{e \tau}\right|$ & $\tau \rightarrow \mu^{+} \mu^{-} \mu^{-}$ & $<2.6 \times 10^{-4}$ \\
\hline
\end{tabular}

Table 1: A collection of all constraints on the elements of $\lambda \equiv v^{2} Y_{E} M_{E}^{-2} Y_{E}^{\dagger} / 8$ from various processes.

These constraints on $\lambda$ will in turn lead to restrictions on the entries for $Y_{E}$ and $M_{E}$.

\section{Acknowledgments}

This work is supported in part by the NSC (grant numbers: NSC-97-2112-M-033-002-MY3 and NSC-99-2811-M-033-013) and in part by the NCTS of Taiwan.

\section{References}

[1] C. K. Chua and S. S. C. Law, Phenomenological constraints on minimally coupled exotic lepton triplets, arXiv:1011.4730 [hep-ph].

[2] K. Nakamura [Particle Data Group], Review of particle physics, J. Phys. G 37, 075021 (2010). 